\title{
ESTIMATIVA DE VALORES ECONOMIZADOS COM A CESSAÇÃO DO TABAGISMO
}

\author{
Cecília Fanha Dornelles ${ }^{1}$ \\ Matheo Augusto Morandi Stumpf ${ }^{2}$ \\ Maki Caroline Nakamura ${ }^{3}$ \\ Samuel Cavalli Kluthcovsky ${ }^{4}$ \\ Bruna Trevisan Vernizi ${ }^{5}$ \\ Erildo Vicente Müller ${ }^{6}$ \\ Ana Cláudia Garabeli Cavalli Kluthcovsky ${ }^{7}$ \\ Recebido em: $21 \mathrm{fev} .2018$ \\ Aceito em: 25 nov. 2018
}

RESUMO: Este estudo objetivou estimar o valor financeiro que o tabagista economizaria ao longo dos anos, caso depositasse em uma aplicação financeira o valor utilizado na compra de cigarros a cada mês. Trata-se de um estudo transversal e quantitativo, com dados de 119 pacientes atendidos em um projeto de extensão universitária, entre $2015 \mathrm{e}$ 2016. Foram analisadas as informações sobre a idade, gênero, data do início do tratamento e número de cigarros consumidos por dia. A partir do valor que seria depositado mensalmente em caderneta de poupança, por paciente, caso deixasse de fumar, foram calculadas as estimativas dos valores brutos que seriam economizados em 10 anos e segundo a expectativa de vida. A comparação entre os gêneros foi realizada pelo teste de Mann-Whitney e o nível de significância foi de $5 \%$. Foi observado que, independente da faixa etária, o valor a ser depositado mensalmente, que corresponderia ao gasto mensal com o cigarro, foi muito semelhante, variando de $R \$ 170,39$ a $R \$ 197,41$. O valor bruto que seria economizado ao longo da vida é considerável, especialmente na faixa etária mais jovem, chegando a mais de dois milhões de reais. Nenhuma variável na comparação entre os gêneros apresentou diferença significativa, demonstrando que o valor depositado mensalmente e economizado ao longo da vida seria semelhante em ambos os grupos. Os resultados obtidos devem ser apresentados à população tabagista servindo como incentivo para a cessação do tabagismo.

Palavras-chave: Tabagismo. Economia. Abandono do uso de tabaco.

\section{ESTIMATION OF VALUES SAVED WITH CESSATION OF SMOKING}

ABSTRACT: This study aim to estimate the financial value that a smoker would save along years if he deposited the value used in the purchase of cigarettes each month in a saving account. Cross-sectional quantitative study, with 119 patients attended in a university extension project, between 2015 and 2016. Age, gender, treatment initiation date and number of cigarettes consumed per day were analysed. From the value that is monthly deposited in savings account, per patient, if the smoker stopped smoking,

\footnotetext{
${ }^{1}$ Acadêmica de Medicina. Universidade Estadual de Ponta Grossa. E-mail: dornellescf@gmail.com.

${ }^{2}$ Acadêmico de Medicina. Universidade Estadual de Ponta Grossa. E-mail: matheoaugusto@hotmail.com.

${ }^{3}$ Acadêmica de Medicina. Universidade Estadual de Ponta Grossa. E-mail: maki_naka_7@hotmail.com.

${ }^{4}$ Acadêmico de Engenharia Mecânica da Universidade Federal do Paraná. E-mail: samuelkluth@gmail.com.

${ }^{5}$ Acadêmica de Medicina. Universidade Federal do Paraná-UFPR. E-mail: bruvernizi@gmail.com.

6 Farmacêutico. Doutor em Saúde Coletiva. Universidade Estadual de Ponta Grossa. E-mail: erildomuller@hotmail.com.

${ }^{7}$ Médica. Doutora em Medicina Interna e Ciências da Saúde. Universidade Estadual de Ponta Grossa. E-mail: anafabio2009@gmail.com.
} 
was calculated an estimative of the gross values that would be saved in 10 years and according to life expectancy. Mann-Whitney test was used for gender comparison and the significance level used was $5 \%$. It was observed that, regardless of the age group, the amount to be deposited monthly, which corresponds to the monthly expenditure with cigarette, was very similar, from $R \$ 170,39$ to $R \$ 197,41$. The gross amount that is saved along life is considerable, especially in the younger age group, reaching more than two million reais. There was no variable in the comparison between the genders that had statistic difference, demonstrating that the value deposited monthly and the value saved calculated by life expectancy were similar in both groups. The results obtained should be disclosed to smokers and serve as another incentive for smoking cessation.

Keywords: Tobacco use disorders. Economics. Tobacco use cessation.

\section{INTRODUÇÃO}

Atualmente o tabagismo é considerado um problema de saúde pública devido ao elevado número de fumantes e à alta mortalidade decorrente do seu uso. O tabagismo está associado ao aumento da mortalidade por doenças cardiovasculares, respiratórias e câncer, sendo que aproximadamente $80 \%$ dos óbitos em adultos com mais de 30 anos ocorreram por esses grupos de causas (Xu et al., 2010).

Além disso, a Organização Mundial da Saúde considera o tabagismo como o principal causador de mortes evitáveis no mundo. Estima-se que até 2030 as mortes atribuíveis ao tabagismo irão diminuir $9 \%$ em países desenvolvidos, entretanto em países em desenvolvimento a OMS estima aumento de 6,8 milhões de mortes evitáveis atribuídas ao uso do tabaco (Kroeff; Mengue, 2010).

O Programa Nacional de Controle do Tabagismo e Outros Fatores de Risco de Câncer, inicialmente intitulado Programa Nacional de Controle do Tabagismo, visa à redução do consumo de tabaco e consequentemente a redução da morbimortalidade por doenças relacionadas a essa substância. Atualmente o programa de controle do tabagismo é considerado como referência mundial. O programa apresenta três grupos de ações centrais: ações educativas, de promoção e apoio à cessação tabágica e de ações legislativas e econômicas. Dentro das últimas ações incluem-se políticas tributárias e de preço a fim de reduzir o consumo de tabaco (Silva et al., 2014). O aumento do preço é uma estratégia para torná-lo um produto menos acessível, principalmente para a população mais jovem (Hwang; Park, 2017).

Observa-se na literatura médica atual poucos trabalhos que avaliam o impacto financeiro pessoal do tabagismo. Assim, o objetivo desse artigo foi estimar os valores em reais que os tabagistas participantes do projeto de extensão "Educando e Tratando o Tabagismo" economizariam ao longo dos anos, caso depositassem em uma aplicação financeira o valor gasto na compra de cigarros a cada mês. 


\section{MATERIAIS E MÉTODOS}

Trata-se de um estudo epidemiológico com delineamento transversal, e abordagem quantitativa. Os dados foram coletados dos prontuários de todos os 119 pacientes atendidos no projeto de extensão "Educando e Tratando o Tabagismo", no período entre 2015 e 2016.

O projeto de extensão "Educando e Tratando o Tabagismo" desenvolvido na Universidade Estadual de Ponta Grossa (UEPG) é vinculado ao Programa Nacional de Controle do Tabagismo do Ministério da Saúde. Possui caráter multidisciplinar, contando com a participação de docentes e acadêmicos dos cursos de graduação em Enfermagem, Medicina e Farmácia. Seus objetivos principais são estimular o abandono do tabagismo e promover educação em saúde em grupos sobre o tabagismo, por meio de abordagens cognitivo-comportamentais e terapia medicamentosa.

Foram analisadas as seguintes informações: idade, gênero, data do início do tratamento e número de cigarros consumidos por dia. O número de cigarros consumidos por dia foi obtido levando-se em consideração a resposta do paciente à pergunta: "quantos cigarros fuma habitualmente por dia?", transcrita para o prontuário.

Foi realizado o cálculo do valor que seria depositado mensalmente em uma caderneta de poupança, caso o paciente parasse de fumar. Para isso multiplicou-se o número de cigarros consumidos por dia e por fumante pelo custo médio de um cigarro em abril de $2016(\mathrm{R} \$ 0,275)$ e o valor encontrado foi multiplicado por 30 dias. O custo médio de um cigarro foi calculado com base nos preços dos cigarros consumidos pelos participantes do projeto. A taxa de rendimento mensal utilizada foi de $0,6581 \%$, correspondente à média dos rendimentos mensais da caderneta de poupança dos anos de 2015 e 2016 (IPARDES, 2017). O início da aplicação foi a partir do primeiro dia de participação no projeto de extensão. O momento final da aplicação levou em consideração a "Tábua Completa de Mortalidade para o total da população brasileira 2015" para homens e mulheres, disponibilizada pelo Instituto Brasileiro de Geografia e Estatística (IBGE, 2017). Essa tábua fornece a expectativa de vida da pessoa a partir da idade atual. Assim, para cada paciente, o intervalo entre a idade no início da participação no projeto e a expectativa de vida fornecida pelo IBGE foi o tempo utilizado para calcular quanto cada paciente economizaria, caso aplicasse o valor mensal que seria gasto com a compra de cigarros. Além disso, foram também calculados os valores economizados em 10 anos, a partir do início da participação no projeto.

As estimativas dos valores economizados ao se aplicar o dinheiro mensal do tabagismo na caderneta de poupança, tendo em vista a expectativa de vida à idade, foram realizados utilizando-se uma fórmula de juro composto, conforme preconizado pelo Banco Central do Brasil (Figura 1) (BCB, 2016).

Frequências absoluta e relativa foram utilizadas para a análise da variável número de sujeitos. Medidas estatísticas descritivas foram utilizadas nas análises da faixa etária, do consumo de cigarros por dia e do valor médio que seria depositado por paciente 
mensalmente.

$$
S_{n}=(1+j) \frac{(1+j)^{n}-1}{j} p
$$

Figura 1 - Fórmula utilizada para cálculo da estimativa do valor que seria economizado.

$\mathrm{Sn}=$ montante ou valor obtido no final; $\mathrm{j}=$ taxa de juro mensal; $p=$ valor de depósito regular; $n=$ número de meses que seriam vividos segundo a expectativa de vida à idade.

A distribuição do número de sujeitos, da média de consumo de cigarros por dia, das estimativas dos valores médios que seriam depositados e valores brutos economizados em 10 anos e segundo expectativa de vida foram apresentados por faixas etárias (19 a 29, 30 a 39,40 a 49,50 a 59 e 60 a 73 anos).

A idade dos pacientes, as estimativas dos valores médios que seriam depositados, os valores brutos economizados em 10 anos e segundo expectativa de vida foram apresentados segundo o gênero. Foi realizada a comparação dessas variáveis entre homens e mulheres pelo teste de Mann-Whitney, após verificação de gaussianidade utilizando-se histogramas e o teste de Kolmogorov-Smirnov.

Os dados foram processados no banco de dados Excel 2010 e a análise estatística foi realizada no programa Statistical Package for Social Science (SPSS), versão 18.0. 0 nível de significância utilizado foi de $5 \%$. O projeto de pesquisa foi aprovado pela Comissão de Ética em Pesquisa da Universidade Estadual de Ponta Grossa com Parecer número 1.055.794, CAAE número 43523515.8.0000.0105.

\section{RESULTADOS}

No total, 119 pacientes foram analisados neste estudo. A idade média dos participantes do projeto de extensão foi de 46,66 anos (DP=13,24), variando de 19 anos e 73 anos, sendo a maioria mulheres (56,3\%). A média de consumo diário de cigarros foi de $22,49(\mathrm{DP}=12,42)$ e o gasto mensal médio com a compra de cigarros foi de $\mathrm{R} \$ 185,52$ $(\mathrm{DP}=102,42)$.

Ao estratificar os indivíduos por faixa etária, verifica-se maior média de consumo de cigarros na faixa mais jovem, entre 19-29 anos, com 23,9 cigarros/dia (Tabela 01). Entretanto, o valor a ser depositado mensalmente, que corresponde ao gasto mensal com o tabaco, é muito semelhante em todas as faixas etárias. Ao se observar o valor bruto total economizado, percebe-se que a cifra é considerável nos jovens (chegando a mais de 2 milhões de reais) e menor na faixa etária mais idosa, haja vista sua expectativa de vida reduzida. 
Tabela 1 - Distribuição do número de sujeitos, da média de consumo de cigarros por dia, das estimativas dos valores médios que seriam depositados e valores brutos economizados em 10 anos e segundo expectativa de vida, por faixa etária, em pacientes que deixassem de fumar a partir do início do tratamento $(\mathrm{N}=119)$.

\begin{tabular}{llllll}
\hline Faixa & $\begin{array}{l}\text { Número } \\
\text { de } \\
\text { etária } \\
\text { em anos } \\
(\%)\end{array}$ & $\begin{array}{l}\text { Média de } \\
\text { consumo de } \\
\text { cigarros/dia }\end{array}$ & $\begin{array}{l}\text { Valor médio } \\
\text { que seria } \\
\text { depositado por } \\
\text { paciente } \\
\text { mensalmente } \\
\text { (em reais) }\end{array}$ & $\begin{array}{l}\text { Estimativa do } \\
\text { valor bruto } \\
\text { economizado } \\
\text { após 10 anos, } \\
\text { por paciente (em } \\
\text { reais) }\end{array}$ & $\begin{array}{l}\text { Estimativa do valor } \\
\text { bruto total } \\
\text { economizado, } \\
\text { considerando a } \\
\text { expectativa de vida, } \\
\text { por paciente (em } \\
\text { reais) }\end{array}$ \\
\hline $19-29$ & $14(11,76)$ & 23,9 & 197,41 & $36.145,44$ & $2.284 .747,77$ \\
$30-39$ & $26(21,85)$ & 20,6 & 170,39 & $31.198,79$ & $783.507,89$ \\
$40-49$ & $20(16,81)$ & 21,2 & 175,30 & $32.099,31$ & $406.796,01$ \\
$50-59$ & $40(33,61)$ & 23,6 & 195,10 & $35.724,65$ & $208.996,52$ \\
$60-73$ & $19(15,97)$ & 22,8 & 188,01 & $34.424,77$ & $97.491,26$ \\
\hline
\end{tabular}

Ao se comparar os gêneros masculino e feminino, observa-se que nenhuma das variáveis analisadas apresentou significância estatística (Tabela 2). Observa-se gastos semelhantes nos grupos e, consequentemente, valores a serem economizados pela aplicação da caderneta de poupança também.

Tabela 2 - Comparação entre homens e mulheres da idade, das estimativas dos valores médios que seriam depositados, dos valores brutos economizados em 10 anos e segundo expectativa de vida, em pacientes que deixassem de fumar a partir do início do tratamento.

\begin{tabular}{|c|c|c|c|}
\hline \multirow[b]{2}{*}{ Variáveis } & Mulheres $(n=67)$ & Homens $(n=52)$ & \multirow[t]{2}{*}{$p^{*}$} \\
\hline & $\begin{array}{l}\text { Média } \\
\text { (Desvio-padrão) } \\
\text { Mediana } \\
\end{array}$ & $\begin{array}{l}\text { Média } \\
\text { (Desvio-padrão) } \\
\text { Mediana }\end{array}$ & \\
\hline Idade em anos & $\begin{array}{l}44,36 \\
(12,65) \\
43,00\end{array}$ & $\begin{array}{l}48,43 \\
(13,50) \\
50,00\end{array}$ & 0,12 \\
\hline $\begin{array}{l}\text { Valor médio que seria } \\
\text { depositado por paciente } \\
\text { mensalmente (em reais) }\end{array}$ & $\begin{array}{l}206,09 \\
(122,31) \\
165,00\end{array}$ & $\begin{array}{l}169,56 \\
(81,26) \\
165,00\end{array}$ & 0,24 \\
\hline $\begin{array}{l}\text { Estimativa do valor bruto } \\
\text { economizado após } 10 \\
\text { anos, por paciente (em } \\
\text { reais) }\end{array}$ & $\begin{array}{l}37.734,85 \\
(22.396,16) \\
30.211,12\end{array}$ & $\begin{array}{l}31.045,31 \\
(14.877,88) \\
30.211,12\end{array}$ & 0,24 \\
\hline $\begin{array}{l}\text { Estimativa do valor bruto } \\
\text { total economizado, } \\
\text { considerando a } \\
\text { expectativa de vida, por } \\
\text { paciente (em reais) }\end{array}$ & $\begin{array}{l}501.384,78 \\
(497.580,00) \\
323.027,87\end{array}$ & $\begin{array}{l}666.175,82 \\
(1.090 .975,30) \\
255.366,84\end{array}$ & 0,45 \\
\hline
\end{tabular}

\footnotetext{
${ }^{\star}$ Teste de Mann-Whitney
} 


\section{DISCUSSÃO}

Poucos estudos avaliam os valores gastos com o tabagismo, sejam mensais ou durante toda a vida. Um estudo realizado por Bazotti et al. (2016) analisou a Pesquisa de Orçamentos Familiares (POF) de 2008-2009 e demonstrou que aproximadamente 10\% da população brasileira apresenta gastos com produtos relacionados ao tabaco. Em geral, a maioria dos pacientes tabagistas possuem baixas renda e escolaridade, idade mais avançada e são predominantemente do sexo masculino. Da renda dos indivíduos, $2,23 \%$ é despendida nestes produtos, correspondendo em média a $R \$ 9,80$ mensais em valores não corrigidos (variando de $\mathrm{R} \$ 0,10$ a $\mathrm{R} \$ 200,00$ ). Porém, algumas observações devem ser feitas. A POF analisou qualquer produto proveniente do tabaco, e não somente cigarros, 0 que explica uma média mensal de gasto menor que a encontrada na presente pesquisa. Além disso, os valores não corrigidos pela inflação e a política governamental de aplicação de novos impostos em produtos derivados do tabaco podem subestimar os verdadeiros gastos absolutos desses pacientes.

De qualquer maneira, é preocupante o achado o predomínio de tabagistas em classes socioeconômicas mais baixas. Os pacientes acabam muitas vezes consumidos por seu vício e gastam mais em cigarros do que com a própria alimentação. De fato, a Fundação Getúlio Vargas, em 2013, afirma que os gastos da população com cigarros têm se mantido nos últimos anos e que há, por mês, comprometimento de $1,2 \%$ da renda com estes produtos contra 0,6\% para compra de arroz e feijão (Agência Brasil, 2017).

Entretanto, dados do Índice Nacional de Preços ao Consumidor Amplo de 2016 monstram que o gasto com o tabagismo caiu, para $1,08 \%$ da renda mensal contra $1,12 \%$ do feijão e arroz (IPCA, 2017). Uma das principais medidas que explicam essa diminuição é o aumento do preço do maço do cigarro, com impostos, medida antitabágica efetiva já bem estabelecida na literatura (Verguet et al., 2017; Golden et al., 2017; Stevens et al., 2017). Com esta medida, os pacientes se sentem mais motivados a cessar o hábito ou reduzir o número de cigarros fumados por dia, além de desestimular o início para os não tabagistas. No Brasil, este tipo de medida reduziu a prevalência de $23,5 \%$ de famílias que gastavam com tabagismo no ano de 2002/2003 para 18,2\% em 2008/2009 (Garcia et al., 2015).

Outro estudo brasileiro, também utilizando a base POF, encontrou porcentagens menores ao avaliar o acometimento da renda mensal com o gasto no hábito tabágico. Pacientes com renda de até $R \$ 400,00$ chegavam a gastar $8,1 \%(R \$ 17,10)$ dela com cigarros mensalmente. Quando a faixa da renda subia de $R \$ 401,00$ a $R \$ 600,00$ a porcentagem do gasto caia para $4,2 \%(R \$ 21,10)$. Pacientes com renda superior a $R \$ 6.000,00$ chegavam a gastar somente $0,6 \%(R \$ 60,60)$ em seu hábito tabágico, ou seja, quanto maior era a renda familiar, menor era a porcentagem gasta com o tabagismo. Este é um dado preocupante haja vista que a maioria dos pacientes fumantes são de baixa renda e gastam, proporcionalmente, mais para manter seu vício. No presente estudo não foi realizada essa análise em relação à renda familiar, porém é um dado importante para ser 
apresentado aos tabagistas, a fim de mostrar o impacto que o vício tem sobre o orçamento mensal, sendo mais um incentivo à cessação do tabagismo (Kroeff; Mengue, 2010).

Outro efeito maléfico do tabagismo é a indisponibilidade de se investir o dinheiro gasto com cigarro em outras atividades ou bens. A cada 5 maços de cigarro consumidos por pessoa ao mês há redução significativa do gasto em outras demandas, especialmente educação e assistência médica. Por outro lado, famílias com ex-tabagistas, no futuro, irão gastar significativamente mais em cuidados médicos do que famílias que nunca tiveram fumantes (Xin et al., 2009).

Deve-se ressaltar que nenhum estudo na literatura médica, até o momento, avaliou os valores que seriam economizados por um paciente tabagista aplicando seu gasto mensal com os cigarros em uma caderneta de poupança. Os resultados apresentados por este trabalho devem ser levados em consideração e subsidiar programas de incentivo de cessação do tabaco. Como observado na Tabela 01, pacientes jovens têm um valor a ser economizado ao longo da vida acima de 2 milhões de reais. Mesmo os pacientes pertencentes a faixa etária mais velha (entre 60-73 anos) apresentam um valor a ser economizado bem considerável, de cerca de 97 mil reais, fato que é mais um incentivo para eles pararem de fumar independentemente da idade. $O$ valor bruto economizado após 10 anos em todas as da faixa etária é de aproximadamente 30 mil reais. Com este valor seria possível ao tabagista adquirir, por exemplo, um carro popular.

White et al. (2005) demonstraram estratégias de economia para pacientes tabagistas. Os autores descrevem que a redução de 3 cigarros ao dia economizava 18 dólares ao mês, contra 6 a 12 dólares ao mês com outras estratégias (como uso de marcas mais baratas, ofertas promocionais, comprar em pacotes com vários maços, entre outras). Isso fortalece a tese de que o dinheiro economizado é sim maior com a cessação total ou parcial do tabagismo.

O programa VIGITEL (vigilância de fatores de risco e proteção para doenças crônicas por inquérito telefônico) de 2016 aponta que a frequência de adultos fumantes foi de $10,2 \%$ nas 26 capitais brasileiras e distrito federal, sendo maior no sexo masculino $(12,7 \%)$ do que no feminino (8,0\%). No total da população, a frequência de fumantes tendeu a ser maior entre os adultos de 45 a 64 anos (BRASIL, 2017).

No nosso estudo, a maioria dos fumantes eram mulheres, essa diferença entre os estudos, pode ser devido ao fato do projeto de extensão ocorrer em horário comercial, favorecendo a participação de donas de casa e aposentados. Apesar disso, foi demonstrado neste estudo que os gastos mensais em cigarros são semelhantes independe do gênero.

Os gastos com saúde em pacientes tabagistas também são maiores do que na população geral. Homens que param de fumar aos 35 anos de idade economizam 7600 euros em gastos de saúde ao longo da vida. Se somado à economia que o paciente teria com sua produtividade (relacionada a aposentadoria precoce, dias afastados do trabalho por comorbidades e mortalidade), o valor chega a 24800 euros ao longo da vida 
(Rasmussen et al., 2005). Logo, neste estudo, supõe-se que o valor a ser economizado ao longo da vida dos pacientes seja ainda maior, pois os gastos com saúde e o impacto na jornada de trabalho não foram levados em conta no cálculo final.

$O$ uso rotineiro de calculadoras (que determinem o gasto que o paciente teria ao longo dos anos com o hábito) deve ser incentivado. O paciente terá um estímulo a mais para cessar o tabagismo, trazendo tanto benefícios financeiros como de saúde em geral a esses indivíduos (Smoke Free, 2017).

Este estudo apresenta algumas limitações. Não foi considerada a expectativa de vida para os tabagistas e sim para população geral. Além disso, por ter sido realizado em centro único, os valores gastos por essa população podem não ser representativos em locais com maços de cigarro mais baratos, por exemplo. É essencial, porém, ressaltar que os dados primários foram coletados pelos próprios pesquisadores e não de base terciária como a POF, fonte dos demais estudos brasileiros publicados.

\section{CONSIDERAÇÕES FINAIS}

O tabagismo é uma doença grave que merece atenção e tratamento adequados. Os resultados aqui apresentados devem ser informados à população em geral e aos tabagistas para incentivar tanto a cessação como ao não início do tabagismo. Espera-se que os pacientes, ao perceberem os valores que poderiam ser economizados ao longo da vida sintam-se mais motivados a cessar este hábito.

\section{REFERÊNCIAS}

Agência Brasil. Consumidor gasta mais com cigarros do que com arroz e feijão. Disponível em: <http://memoria.ebc.com.br/agenciabrasil/noticia/2013-05-31/consumidorgasta-mais-com-cigarros-do-que-com-arroz-e-feijao>. Acesso em: 07 ago. 2017.

Banco Central do Brasil - BCB. Calculadora do cidadão. Disponível em: <https://www3.bcb.gov.br/CALCIDADAO/publico/exibirMetodologiaAplicacaoDepositosRe gulares.do?method=exibirMetodologiaAplicacaoDepositosRegulares $>$. Acesso em: 18 abr. 2016.

Bazotti, A. et al. Tabagismo e pobreza no Brasil: uma análise do perfil da população tabagista a partir da POF 2008-2009. Ciência \& Saúde Coletiva, v. 21, n. 1, p. 45-52, 2016.

BRASIL. Ministério da Saúde. Vigilância de fatores de risco e proteção para doenças crônicas por inquérito telefônico: Vigitel 2016. Brasília: Ministério da Saúde; 2017. Disponível em:

$<$ http://www1.inca.gov.br/inca/Arquivos/comunicacao/vigitel_2016_jun17.pdf>. Acesso em: 12 jan. 2018.

Garcia, L. P. et al. A política antitabagismo e a variação dos gastos das famílias 
brasileiras com cigarro: resultados das Pesquisas de Orçamentos Familiares, 2002/2003 e 2008/2009. Cadernos de Saúde Pública, v. 31, n. 9, p. 1894-1906, 2015.

Golden, S. D. et al. Comparing projected impacts of cigarette floor price and excise tax policies on socioeconomic disparities in smoking. Tobacco Control, v. 25, suppl. 1, p. i6066, 2016. Disponível em:< https://www.ncbi.nlm.nih.gov/pmc/articles/PMC5099216/>. Acesso em: 07 nov. 2017.

Hwang, J. H.; Park, S. W. Is there differential responsiveness to a future cigarette price increase depending on adolescents' source of cigarette access? Medicine (Baltimore), v. 96, n. 26, e7311, 2017. Disponível em:<

https://www.ncbi.nlm.nih.gov/pmc/articles/PMC5500062/>. Acesso em: 07 nov. 2017.

Índice Nacional de Preços ao Consumidor Amplo (IPCA). Disponível em:

<https://www.portalbrasil.net/ipca.htm>. Acesso em: 07 ago. 2017.

Instituto Brasileiro de Geografia e Estatística - IBGE. Tábuas Completas de Mortalidade 2015. Disponível em:

<2015http://www.ibge.gov.br/home/estatistica/populacao/tabuadevida/2015/default.shtm>. Acesso em: 12 abr. 2017.

Instituto Paranaense de Desenvolvimento Econômico e Social - IPARDES. Rendimentos da Caderneta de Poupança - Brasil. Disponível em:

<http://www.ipardes.gov.br/pdf/indices/poupanca.pdf>. Acesso em 18 abr. 2017.

Kroeff, L. R.; Mengue, S. S. Análise dos gastos individuais com tabagismo a partir da Pesquisa de Orçamentos Familiares de 2002-2003. Cadernos de Saúde Pública, v. 26, n. 12, p. 2334-2342, 2010.

Rasmussen, S. R. et al. The total lifetime health cost savings of smoking cessation to society. European Journal of Public Health, v. 15, n. 6, p. 601-606, 2005.

Silva, S. T. et al. Combate ao Tabagismo no Brasil: a importância estratégica das ações governamentais. Ciência \& Saúde Coletiva, v. 19, n. 2, p. 539-552, 2014.

Smoke Free. Disponível em: <https://smokefree.gov/how-much-will-you-save>. Acesso em: 07 ago. 2017.

Stevens, V. L. et al. A Prospective Cohort Study of Cigarette Prices and Smoking Cessation in Older Smokers. Cancer Epidemiology, Biomarkers and Prevention, v. 26, n. 7, p. 1071-1077, 2017.

Verguet, S. et al. The consequences of tobacco tax on household health and finances in rich and poor smokers in China: an extended cost-effectiveness analysis. Lancet Global Health, v. 3, n. 4, e206-2016, 2015. Disponível em:< http://www.thelancet.com/journals/langlo/article/PIIS2214-109X(15)70095-1/abstract>. Acesso em: 07 nov. 2017.

White, V. M. et al. How do smokers control their cigarette expenditures? Nicotine Tobacco Research, v. 7, n. 4, p. 625-635, 2005.

Xin, Y. et al. The impact of smoking and quitting on household expenditure patterns and 
medical care costs in China. Tobacco Control, v. 18, n. 2, p. 150-155, 2009.

$\mathrm{Xu}, \mathrm{X}$. et al. Annual healthcare spending attributable to cigarette smoking: an update. American Journal of Preventive Medicine, v. 48, n. 3, p. 326-333, 2015. 